\title{
Neural Dysregulation in Post-Covid Fatigue
}

\author{
Anne M.E. Baker ${ }^{1 \#}$, Natalie J. Maffitt ${ }^{1 \#}$, Alessandro Del Vecchio ${ }^{2}$, Katherine M. \\ McKeating ${ }^{1}$, Mark R. Baker ${ }^{1}$, Stuart N. Baker ${ }^{1}$ and Demetris S. Soteropoulos ${ }^{1 *}$
}

\author{
1: Faculty of Medical Sciences, Newcastle University, UK. \\ 2: Department Artificial Intelligence in Biomedical Engineering, Friedrich-Alexander University \\ Erlangen-Nürnberg, Germany. \\ \#: equal contribution \\ *: corresponding author
}

Following infection from SARS-CoV-2, a substantial minority of people develop lingering after-effects known as 'long COVID'. Fatigue is a common complaint with substantial impact on daily life, but the neural mechanisms behind post-COVID fatigue remain unclear. We recruited volunteers with self-reported fatigue after a mild COVID infection and carried out a battery of behavioural and neurophysiological tests assessing the central, peripheral and autonomic nervous systems. In comparison to age and gender matched volunteers without fatigue, we show underactivity in specific cortical circuits, dysregulation of autonomic function, and myopathic change in skeletal muscles. Cluster analysis revealed no subgroupings, suggesting post-COVID fatigue is a single entity with individual variation, rather than a small number of distinct syndromes. These abnormalities on objective tests may indicate novel avenues for principled therapeutic intervention, and could act as fast and reliable biomarkers for diagnosing and monitoring the progression of fatigue over time.

Most people infected with SARS-CoV-2 do not require hospitalisation. However, even after a mild infection, a minority develop symptoms that linger for weeks or months (long COVID). Persistent fatigue, where everyday actions become laborious, is one of the more commonly reported aftereffects $^{1}$ and can have a substantial impact on the quality of life and productivity of sufferers ${ }^{2-4}$. At the time of publication $\sim 2 \%$ of the UK population are experiencing long COVID; $>50 \%$ report fatigue as their primary symptom ${ }^{5}$.

Fatigue appears to be a multi-system pathology, with immunological, metabolic and hormonal anomalies. These presumably generate changes in neurological function ${ }^{6}$ which lead to feelings of weakness and actions being more effortful. Changes could occur at many loci - for example, reduced activation of central motor circuits or impaired muscle function could lead to weakness; upregulated sensory feedback could increase sense of effort. To date no studies have investigated deficits in specific neural circuits with post-covid fatigue (pCF).

To understand the neural mechanisms behind $\mathrm{pCF}$, we exploited a wide range of wellcharacterised non-invasive behavioural and neurophysiological tests. Thirty seven volunteers suffering from pCF after a SARS-CoV-2 infection which had not required hospitalization attended a laboratory visit 6-26 weeks after diagnosis. Fifty two controls were age and sex matched to the pCF cohort. Based on the published relative incidence of SARS-CoV-2 variants in the UK, we estimate $\sim 83 \%$ of our pCF cohort had the Alpha variant (see Supplementary Fig. 1). During the $\sim 4$ hour lab visit, a series of behavioural and neurophysiological examinations assessed specific neural systems. These tests yielded 35 measures (33 relating to the state of several components of the nervous system, plus blood oxygen saturation and tympanic temperature; Supplementary Table 1), summarised graphically in Fig. 1. Figure 2A summarises the differences between pCF 
medRxiv preprint doi: https://doi.org/10.1101/2022.02.18.22271040; this version posted February 21, 2022. The copyright holder for this preprint

(which was not certified by peer review) is the author/funder, who has granted medRxiv a license to display the preprint in perpetuity. It is made available under a CC-BY-NC-ND 4.0 International license .

and control groups using normalised ( $Z$ scored) measures; details of tests, calculations and statistical comparisons are given in Supplementary Notes and Supplementary Table 2.

Fatigue could arise from a reduced ability to generate force; a given movement would then require stronger voluntary drive and perceived effort would rise. Changes could arise in muscles themselves ${ }^{7}$, due to a weakened connection from motoneurons to muscles fibres ${ }^{8}$, or because motoneurons are less excitable. We found that maximal grip strength was not significantly reduced in pCF, suggesting no deficit in force production for brief contractions. The efficacy of transmission at the neuromuscular junction, and intrinsic motoneuron excitability assessed with multiple measures, were not significantly different between our twocohorts. However, when we tested changes during a prolonged maximal contraction, we found pCF subjects had an increased level of peripheral fatigue (maximal electrically-evoked twitch reduced after sustained contraction to $48.5 \pm 5.3 \%$ in pCF vs $67.1 \pm 3.6 \%$ in controls, mean \pm SEM, $p<0.0033$ ). This suggests that pCF produces metabolic changes in muscle fibres leading to reduced force output after prolonged activity.

Voluntary activation of muscles relies on command signals from motor areas of the cortex; the state of cortical circuits has been linked to perception of effort and force output during fatiguing contractions $^{9,10}$. We used transcranial magnetic stimulation (TMS) to assess the function of primary motor cortex. Intracortical facilitation (ICF) was significantly lower in pCF (conditioned motor evoked potential relative to unconditioned $170.6 \pm 13 \%$ in pCF vs $258.3 \pm 19.7 \%$ in controls, $\mathrm{p}<0.001$ ), suggesting reduced cortical excitability. By contrast, multiple measures of cortical inhibition showed no significant differences. Disturbances in sensory feedback processing have been previously hypothesised to contribute to an increased perception of effort ${ }^{11}$, but tests of sensory function revealed no significant differences, suggesting that this is unlikely to be a contributing factor in pCF.

Autonomic dysregulation is often associated with fatigue in other conditions ${ }^{12,13}$ and recent studies reported autonomic dysregulation after SARS-CoV-2 infection ${ }^{14-17}$ (although not universally ${ }^{18}$ ). We found an increased resting heart rate in PCF $(74.8 \pm 1.9$ vs $67.7 \pm 3.6$ beats $/ \mathrm{min}, p<0.0017)$. Other measures of autonomic function (tympanic temperature, heart rate variability ( $p N N 50$ ) and galvanic skin response habituation) also differed between cohorts, and although significant at $p<0.05$ level, they did not pass correction for multiple comparisons. This points towards an increased vagal (relative to sympathetic) tone, suggesting pCF subjects suffer from a degree of dysautonomia.

At least three neurophysiological measures were different between $\mathrm{pCF}$ and controls; there was also a significant difference in blood oxygen saturation $(95.3 \pm 1.9 \%$ vs $97.2 \pm 1.5 \%, P=0.00002)$. We investigated whether these dysregulations occurred in the same people, or whether pCF could be sub-divided into two or more different syndromes. K-means clustering followed by gap analysis revealed that the optimal cluster number was one (Fig. 2B), regardless of whether we included all metrics, or the four which were significantly different after correction for multiple comparisons. Dysregulations thus appear to vary independently across the pCF population, rather than being clustered in particular subsets.

For the metrics which failed to reach significance individually, it was important to determine if they could still distinguish between pCF and controls in combination. We used a machine learning approach to classify participants as pCF or control, based on the multivariate data. Out of 33 available neurophysiological or behavioural metrics, repeated runs of the classifier determined the optimal feature number to maximise classifier accuracy. This had a mode of six (Fig. 2C). We then ran repeated classifications locked to six features, and counted how often a given measure was used (Fig. 2D). With this approach, the mean classification accuracy was $70 \%$ (SD $3.6 \%$ ). In addition to the three neurophysiological features which were individually significantly different as described above, additional frequently selected metrics were the habituation of the galvanic skin response to a startling stimulus and heart rate variability (autonomic measures), visual reaction times and StartReact effect (multimodal measures of sensorimotor function), and another measure 
medRxiv preprint doi: https://doi.org/10.1101/2022.02.18.22271040; this version posted February 21, 2022. The copyright holder for this preprint

(which was not certified by peer review) is the author/funder, who has granted medRxiv a license to display the preprint in perpetuity.

It is made available under a CC-BY-NC-ND 4.0 International license .

of cortical excitability. All bar one of these additional measures had individual significance levels $\mathrm{P}<0.05$, but had failed the correction for multiple comparisons. This analysis suggests that there is little redundancy between measures and each captures a separate dimension of dysregulation in pCF.

The rapid development of vaccines against SARS-CoV-2 means that despite the evolution of variants, the majority of people in the UK are now largely protected from adverse short-term effects. Immunity also offers significant, but incomplete, protection from lingering sequelae ${ }^{19}$. pCF is thus likely to grow less rapidly than it has recently; nevertheless, the number still suffering remains staggering. Current estimates suggest $\sim 1 \%$ of the population have lasting fatigue, with enormous economic and social cost.

There is thus an urgent need for better understanding of the physiological mechanisms of long COVID, including pCF. Biomarker identification ${ }^{20}$ would allow an objective diagnosis; this is a syndrome which remains beset by uncertainties over the organic versus psychological basis of the experiences of sufferers. If abnormalities precede or appear early after an infection, they could be used to predict who is most likely to develop long term sequelae. Finally, knowledge of which neural systems are compromised in PCF could guide development of rational therapeutics. Our results provide evidence of dysregulation in all three main divisions of the nervous system, using tests which are straightforward to administer and could easily be incorporated in future trials to assess and help treat pCF.

\section{References}

1 Blomberg, B. et al. Long COVID in a prospective cohort of home-isolated patients. Nature medicine 27, 1607-1613, doi:10.1038/s41591-021-01433-3 (2021).

2 McCrone, P., Darbishire, L., Ridsdale, L. \& Seed, P. The economic cost of chronic fatigue and chronic fatigue syndrome in UK primary care. Psychol Med 33, 253-261, doi:10.1017/s0033291702006980 (2003).

3 Collin, S. M. et al. The impact of CFS/ME on employment and productivity in the UK: a cross-sectional study based on the CFS/ME national outcomes database. BMC Health Serv Res 11, 217, doi:10.1186/1472-6963-11-217 (2011).

4 Cullinan, J., Ni Chomhrai, O., Kindlon, T., Black, L. \& Casey, B. Understanding the economic impact of myalgic encephalomyelitis/chronic fatigue syndrome in Ireland: a qualitative study. HRB Open Res 3, 88, doi:10.12688/hrbopenres.13181.1 (2020).

5 Office for National Statistics. Prevalence of ongoing symptoms following coronavirus (COVID-19) infection in the UK. (2022). $<$ https://www.ons.gov.uk/peoplepopulationandcommunity/healthandsocialcare/conditionsan ddiseases/bulletins/prevalenceofongoingsymptomsfollowingcoronaviruscovid19infectioninth euk/6january2022>.

6 Dantzer, R. Neuroimmune Interactions: From the Brain to the Immune System and Vice Versa. Physiological reviews 98, 477-504, doi:10.1152/physrev.00039.2016 (2018).

7 Agergaard, J. et al. Myopathic changes in patients with long-term fatigue after COVID-19. Clin Neurophysiol 132, 1974-1981, doi:10.1016/j.clinph.2021.04.009 (2021).

8 Alekseeva, T. M. et al. Fatigue in patients with myasthenia gravis. J Neurol 265, 23122321, doi:10.1007/s00415-018-8995-4 (2018).

9 Hunter, S. K., McNeil, C. J., Butler, J. E., Gandevia, S. C. \& Taylor, J. L. Short-interval cortical inhibition and intracortical facilitation during submaximal voluntary contractions changes with fatigue. Exp Brain Res 234, 2541-2551, doi:10.1007/s00221-016-4658-9 (2016).

10 Thickbroom, G. W. et al. Central motor drive and perception of effort during fatigue in multiple sclerosis. J Neurol 253, 1048-1053, doi:10.1007/s00415-006-0159-2 (2006).

11 De Doncker, W., Dantzer, R., Ormstad, H. \& Kuppuswamy, A. Mechanisms of poststroke fatigue. J Neurol Neurosurg Psychiatry 89, 287-293, doi:10.1136/jnnp-2017-316007 (2018).

12 Davies, K. \& Ng, W. F. Autonomic Nervous System Dysfunction in Primary Sjogren's Syndrome. Front Immunol 12, 702505, doi:10.3389/fimmu.2021.702505 (2021). 
medRxiv preprint doi: https://doi.org/10.1101/2022.02.18.22271040; this version posted February 21, 2022. The copyright holder for this preprint

(which was not certified by peer review) is the author/funder, who has granted medRxiv a license to display the preprint in perpetuity.

It is made available under a CC-BY-NC-ND 4.0 International license .

13 Pagani, M. \& Lucini, D. Chronic fatigue syndrome: a hypothesis focusing on the autonomic nervous system. Clin Sci (Lond) 96, 117-125 (1999).

14 Leitzke, M., Stefanovic, D., Meyer, J. J., Schimpf, S. \& Schonknecht, P. Autonomic balance determines the severity of COVID-19 courses. Bioelectron Med 6, 22, doi:10.1186/s42234020-00058-0 (2020).

15 Pan, Y. et al. Alteration of Autonomic Nervous System Is Associated With Severity and Outcomes in Patients With COVID-19. Frontiers in physiology 12, 630038, doi:10.3389/fphys.2021.630038 (2021).

16 Kurtoglu, E. et al. Altered cardiac autonomic function after recovery from COVID-19. Ann Noninvasive Electrocardiol 27, e12916, doi:10.1111/anec.12916 (2022).

17 Mol, M. B. A. et al. Heart-rate-variability (HRV), predicts outcomes in COVID-19. PLoS One 16, e0258841, doi:10.1371/journal.pone.0258841 (2021).

18 Townsend, L. et al. Fatigue following COVID-19 infection is not associated with autonomic dysfunction. PLoS One 16, e0247280, doi:10.1371/journal.pone.0247280 (2021).

19 Kuodi, P. et al. Association between vaccination status and reported incidence of postacute COVID-19 symptoms in Israel: a cross-sectional study of patients tested between March 2020 and November 2021. medRxiv, 2022.2001.2005.22268800, doi:10.1101/2022.01.05.22268800 (2022).

20 Phetsouphanh, C. et al. Immunological dysfunction persists for 8 months following initial mild-to-moderate SARS-CoV-2 infection. Nat Immunol, doi:10.1038/s41590-021-01113-x (2022). 

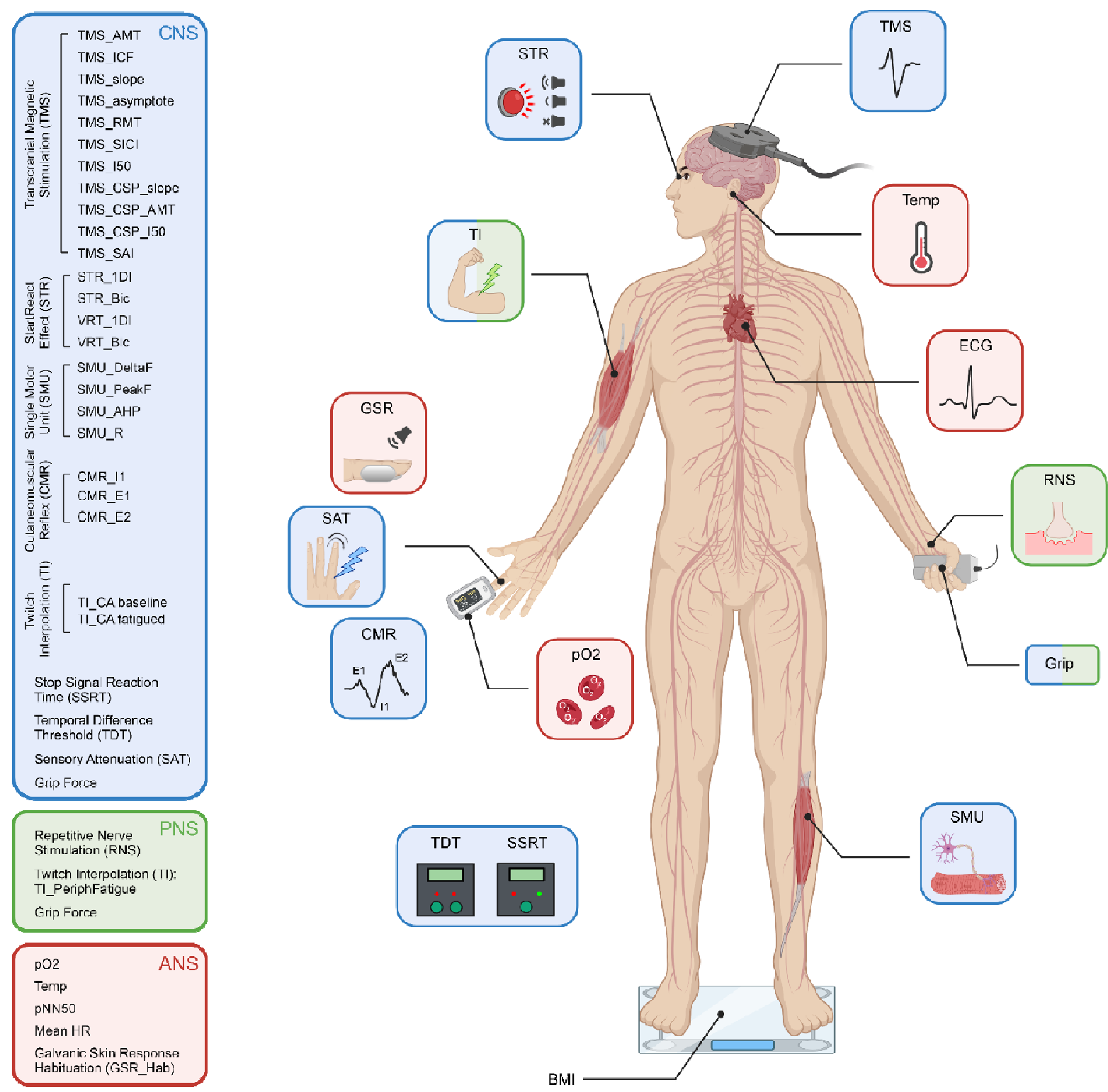

Figure 1. Schematic representation of the different tests performed, colour coded according to which components of the central, peripheral and autonomic nervous systems (CNS, PNS, ANS) they assessed. TMS, transcranial magnetic stimulation; ECG, electrocardiogram; RNS, repetitive nerve stimulation; SMU, single motor unit recording; BMI, body mass index; TDT, temporal difference threshold; SSRT, stop signal reaction time; pO2, blood oxygen saturation; CMR, cutaneomuscular reflex; SAT, sensory attenuation with movement; GSR, habituation of the galvanic skin response to loud sound; TI, twitch interpolation, STR, StartReact effect. 
medRxiv preprint doi: https://doi.org/10.1101/2022.02.18.22271040; this version posted February 21, 2022. The copyright holder for this preprint (which was not certified by peer review) is the author/funder, who has granted medRxiv a license to display the preprint in perpetuity.

It is made available under a CC-BY-NC-ND 4.0 International license .

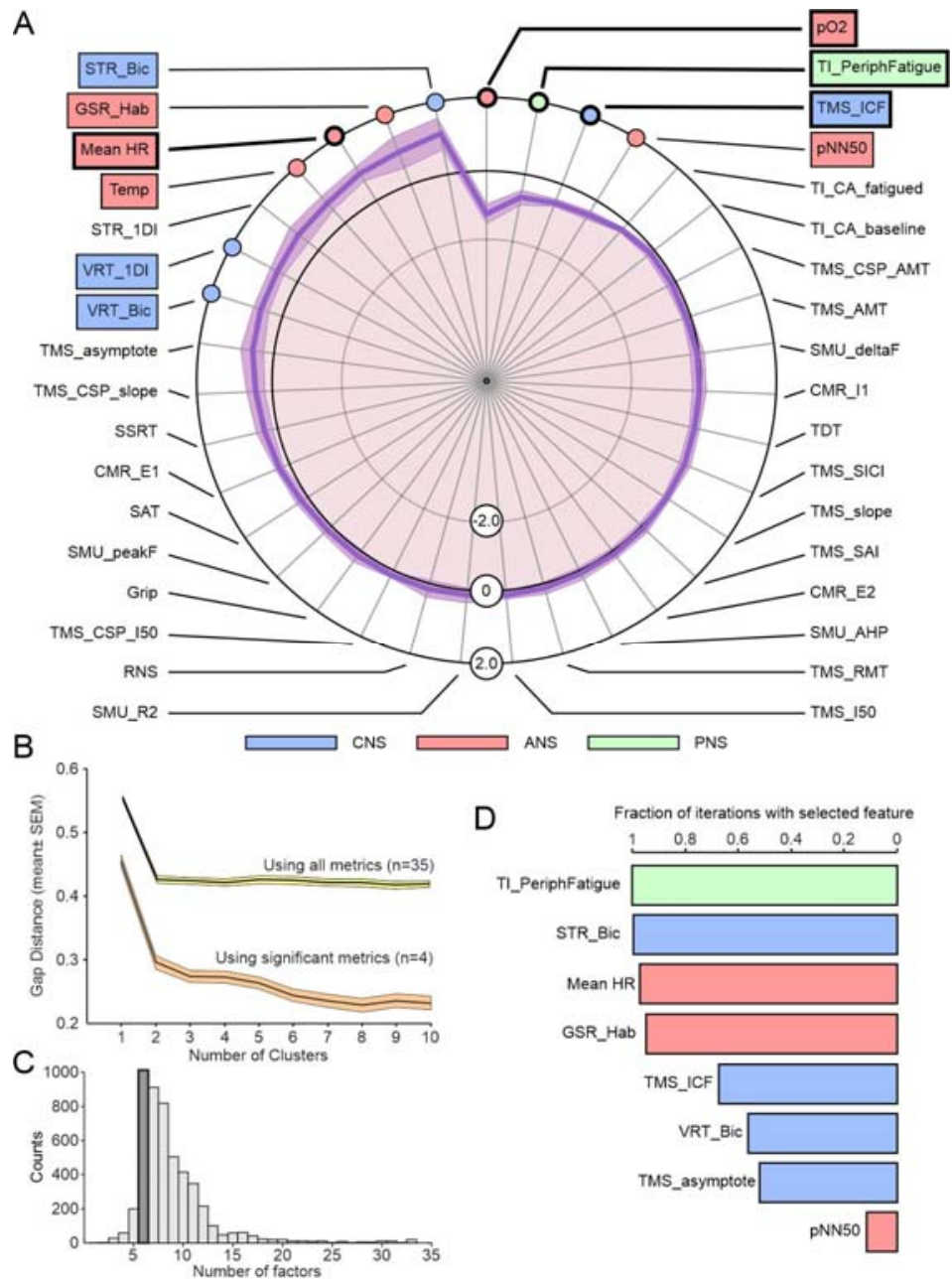

Figure 2. A, results from the tests outlined in Fig. 1, normalised as $Z$ scores (difference between pCF and control subjects, relative to SD). Measures highlighted with coloured boxes were individually significantly different between pCF and controls $(P<0.05)$; for those with thicker lines, significance passed the correction for multiple comparisons. B, gap analysis of number of clusters in the multivariate dataset from pCF subjects. C, number of factors chosen by a machine learning algorithm to maximise classification of pCF versus control subjects. D, fraction of iterations of classification algorithm, with feature number fixed to six, which included different features. Plot has been truncated to show the most common eight features. 